\title{
Acetaminophen: Dose-Dependent Drug Hepatotoxicity and Acute Liver Failure in Patients
}

\author{
Hartmut Jaeschke \\ Department of Pharmacology, Toxicology and Therapeutics, University of Kansas Medical Center, \\ Kansas City, Kans., USA
}

\section{Key Words}

Acetaminophen hepatotoxicity · Acute liver failure ·

Biomarkers · Innate immune response $\cdot$ Mitochondrial dysfunction

\begin{abstract}
Background: Drug-induced liver injury is a rare but serious clinical problem. A number of drugs can cause severe liver injury and acute liver failure at therapeutic doses in a very limited number of patients $(<1: 10,000)$. This idiosyncratic drug-induced liver injury, which is currently not predictable in preclinical safety studies, appears to depend on individual susceptibility and the inability to adapt to the cellular stress caused by a particular drug. In striking contrast to idiosyncratic drug-induced liver injury, drugs with dose-dependent hepatotoxicity are mostly detected during preclinical studies and do not reach the market. One notable exception is acetaminophen (APAP, paracetamol), which is a safe drug at therapeutic doses but can cause severe liver injury and acute liver failure after intentional and unintentional overdoses. Key Messages: APAP overdose is responsible for more acute liver failure cases in the USA or UK than all other etiologies combined. Since APAP overdose in the mouse represents a model for the human pathophysiology, sub-
\end{abstract}

stantial progress has been made during the last decade in understanding the mechanisms of cell death, liver injury and recovery. More recently, emerging evidence based on mechanistic biomarker analysis in patients and studies of cell death in human hepatocytes suggests that most of the mechanisms discovered in mice also apply to patients. The rapid development of $\mathrm{N}$-acetylcysteine as an antidote against APAP overdose was based on the early understanding of APAP toxicity in mice. However, despite the efficacy of $\mathrm{N}$-acetylcysteine in patients who present early after APAP overdose, there is a need to develop intervention strategies for late-presenting patients. Conclusions: The challenges related to APAP toxicity are to better understand the mechanisms of cell death in order to limit liver injury and prevent acute liver failure, and also to develop biomarkers that better predict as early as possible who is at risk for developing acute liver failure with poor outcome.

(c) 2015 S. Karger AG, Basel

\section{Introduction}

Drug hepatotoxicity, although a relatively rare event, is the leading cause of acute liver failure in the clinic, accounting for more than $60 \%$ of all cases in the USA [1].

\section{KARGER 125}

(C) 2015 S. Karger AG, Basel

0257-2753/15/0334-0464\$39.50/0

E-Mail karger@karger.com

www.karger.com/ddi
Dr. Hartmut Jaeschke

Department of Pharmacology, Toxicology and Therapeutics

University of Kansas Medical Center, 3901 Rainbow Boulevard, MS 1018

Kansas City, KS 66160 (USA)

E-Mail hjaeschke@kumc.edu 
A general classification considers drug toxicity as either idiosyncratic or dose dependent. Idiosyncratic drug-induced liver injury is one of the biggest concerns with the development of new drugs as predictable animal models or in vitro tests are not available, and the limited number of patients in clinical trials generally does not allow the detection of delayed, mostly immune-based toxicity that occurs in less than 1 in 10,000 patients [2]. On the other hand, a considerable effort is being made to detect dosedependent drug toxicity in preclinical testing and during clinical trials. Due to the use of numerous in vitro and in vivo approaches, dose-dependent direct toxicity is reliably detectable and, generally, dose-dependent hepatotoxins are eliminated early in the drug development process. However, one of the most used pain medications worldwide, acetaminophen (APAP; paracetamol), is a classical dose-dependent hepatotoxin that is responsible for almost 50\% of all acute liver failure cases in the USA, the UK and many Western countries [1]. Therefore, this review will focus on the recent progress made in the understanding of the pathophysiology of APAP hepatotoxicity in patients and the development of biomarkers that can predict whether a patient will spontaneously recover or need a liver transplant to survive.

\section{Mechanisms of APAP Hepatotoxicity}

\section{Clinical Aspects of APAP Overdose}

APAP is a safe drug at therapeutic doses of $\leq 4 \mathrm{~g}$ per day for an adult. Extensive literature reviews suggest that even susceptible people such as alcoholics are unlikely to suffer adverse effects from therapeutic doses of APAP [3]. However, an overdose can cause severe liver injury and even acute liver failure $[4,5]$. Suicide attempts are a frequent cause of exposure to a single, high overdose of APAP, but unintentional, cumulative overdosing is an increasing problem. APAP is not only found in specific pain medications, but is also present in sleeping aids, medicine for colds and numerous other over-the-counter drugs. Thus, patients taking multiple drugs may inadvertently exceed recommended doses of APAP. Unintentional overdosing frequently has a poorer outcome because these patients generally present later than patients who attempted suicide [4].

The only clinically approved antidote against APAPinduced liver injury is $\mathrm{N}$-acetylcysteine (NAC), which is most effective when administered within $8 \mathrm{~h}$ of the overdose [4]. Although NAC is still beneficial even after $24 \mathrm{~h}$, the efficacy is substantially diminished [6]. NAC is a pre- cursor for glutathione (GSH) synthesis and acts through facilitating scavenging of the reactive metabolite $\mathrm{N}$-acetyl-p-benzoquinone imine (NAPQI) during the metabolism phase [7]. At later time points, GSH assists in scavenging reactive oxygen in mitochondria and excess NAC is converted to Krebs cycle intermediates and supports mitochondrial energy metabolism [8]. Delayed treatment with NAC increases the risk for acute liver failure. However, in contrast to idiosyncratic drug toxicity, APAP-induced liver injury and liver failure has a relatively high survival rate. Nevertheless, due to the widespread use of the drug, APAP overdose is by far the most frequent cause of acute liver failure of any etiology in the USA and the UK [1]. Thus, the major challenges related to APAP overdose are: (1) improving the understanding of the mechanisms of toxicity and regeneration in order to develop therapeutic interventions that reduce the severity of the disease and prevent acute liver failure, and (2) developing biomarkers to predict as early as possible after admission which patient is likely to recover and which will need a transplant to survive.

\section{Mechanisms of APAP-Induced Liver Injury in Preclinical Studies}

Shortly after the first report on APAP hepatotoxicity in humans, a mouse model was developed that showed a similar degree of liver injury as observed in patients [9]. The early experiments demonstrated the formation of a reactive metabolite, later suggested to be NAPQI, which can be scavenged by GSH [9]. However, the excess formation of NAPQI after an overdose causes reactions with protein sulfhydryl groups (protein adduct formation) and toxicity [9]. This early insight into the mechanism led to the development of NAC.

Subsequent studies demonstrated that APAP protein adducts formation per se do not directly cause cell death because no critical targets could be identified [10]. Instead, protein adducts, especially in mitochondria, seem to disturb the electron transport chain and generate an oxidant stress and peroxynitrite in mitochondria [11], which eventually triggers the mitochondrial permeability transition (MPT) pore opening and collapse of the membrane potential [12] (fig. 1). In addition, formation of a Bax pore and later mitochondrial matrix swelling leads to release of intermembrane proteins, especially endonuclease $\mathrm{G}$ and apoptosis-inducing factor, both of which translocate to the nucleus and cause extensive DNA fragmentation $[10,11]$. The combination of massive mitochondrial dysfunction and nuclear disintegration leads to cellular necrosis [13]. 


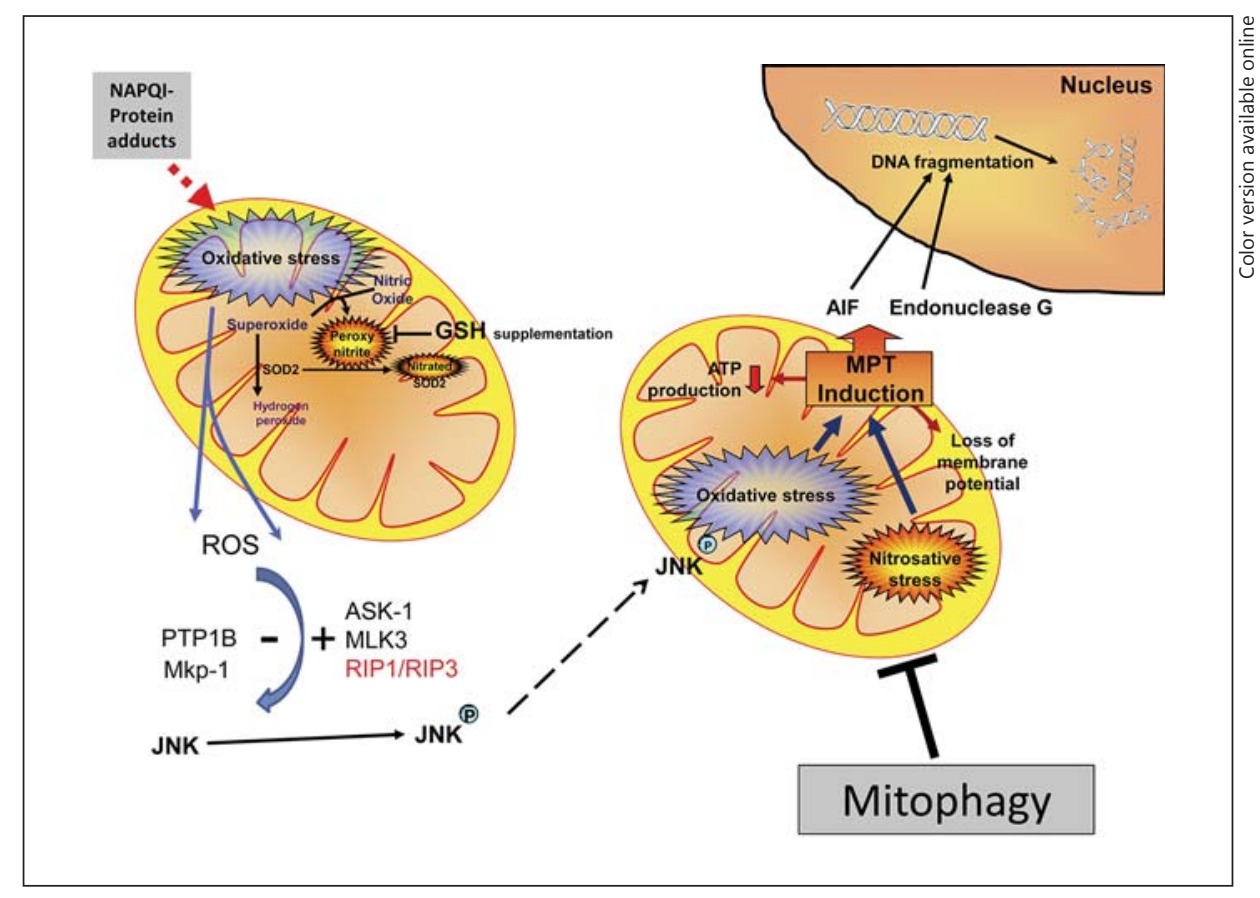

Fig. 1. Mechanisms of APAP-induced necrotic cell death. The P450-dependent metabolism of APAP results in the formation of the reactive intermediate NAPQI, which forms mitochondrial protein adducts and induces a mitochondrial oxidative stress. Superoxide derived from the mitochondrial electron transport chain reacts with nitric oxide resulting in the production of peroxynitrite. Superoxide can be metabolized by superoxide dismutase 2 (SOD2) and converted to hydrogen peroxide, though peroxynitrite can inactivate SOD2 by nitration. The initial mitochondrial oxidative stress can also activate the MAP kinase JNK by multiple pathways (redox-sensitive kinases), resulting in its phosphoryla- tion and translocation to the mitochondria. Mitochondrial JNK translocation amplifies the mitochondrial oxidant stress, which subsequently leads to activation of the MPT pore opening, translocation of mitochondrial proteins such as apoptosis-inducing factor (AIF) and endonuclease $\mathrm{G}$ to the nucleus. This then results in DNA fragmentation and finally oncotic necrosis. Mitophagy can counteract the process by removing damaged mitochondria. ASK1 = Apoptosis signal-regulating kinase 1 ; Mkp-1 = mitogen-activated protein kinase phosphatase $1 ;$ MLK3 = mixed-lineage kinase 3 ; PTP1B = protein tyrosine phosphatase $1 \mathrm{~B}$. Adapted from Jaeschke et al. [11].
More recently, it was recognized that the initial oxidant stress was insufficient to trigger the MPT. Instead, an amplification loop is necessary that involves the activation of various redox-sensitive MAP kinases, including apoptosis signal-regulating kinase 1 and mixed-lineage kinase 3, which ultimately causes the activation of c-Jun $\mathrm{N}$-terminal kinase $1 / 2(\mathrm{JNK})$ and translocation of $\mathrm{p}$-JNK to mitochondria [14] (fig. 1). Upon interaction of p-JNK with an anchor protein in the outer mitochondrial membrane [14], the mitochondrial oxidant stress is amplified [15]. Together with lysosomal iron, which enters mitochondria through the $\mathrm{Ca}^{2+}$ uniporter, the oxidant stress triggers the MPT [16]. Thus, in the mouse model, the JNK loop is critical for the pathophysiology. Recently, the importance of additional kinases, such as receptor-interacting protein kinase (RIP) 1 and RIP3, has been shown in APAP hepatotoxicity $[17,18]$. Whether these kinases act through JNK or other pathways remains unclear. RIP3 has been shown to promote mitochondrial fission and oxidant stress [17]. Ultimately, all signaling pathways lead to cell necrosis (necroptosis) [13, 17]. Apoptosis is not a relevant cell death mechanism of APAP-induced liver injury; there is no evidence for caspase activation and highly potent caspase inhibitors have no effect on the injury $[13,19,20]$.

In addition to the pro-cell death signaling pathways, adaptive defense mechanisms also affect the pathophysiology. Autophagy is a process where damaged organelles are being enveloped with lipid membranes (autophagosomes), which then fuse with lysosomes for degradation [21]. APAP overdose activates autophagy of damaged mitochondria (mitophagy), which can limit liver injury [22] (fig. 1). Mitophagy can affect cell death around the outer area of necrosis where the insult is not too overwhelming 
[23]. Beyond autophagy, the unfolded protein response (ER stress) can be activated [24], but the pathophysiological relevance remains controversial. Furthermore, adaptive responses can be beneficial, such as the induction of heat shock proteins [25], metallothionein [26] or antioxidant enzymes, e.g. glutamate cysteine ligase, which is the rate-limiting enzyme for hepatic GSH synthesis. In fact, the lower susceptibility of female mice to APAP overdose is thought to be mainly caused by the more robust induction of glutamate cysteine ligase and faster recovery of GSH levels [27]. Overall, for all experiments a number of pro-cell death pathways and adaptive defense mechanisms need to be considered.

\section{Mechanisms of APAP-Induced Liver Injury in the Human Liver}

Although most of the known mechanisms of APAPinduced liver injury were discovered in a rodent model, the important question is how relevant these mechanisms are for patients. Similar to mouse hepatocytes, the initial response of primary human hepatocytes or metabolically competent hepatoma cells (HepaRG cells) to high APAP levels is rapid GSH depletion and protein adduct formation, including mitochondrial adducts $[28,29]$. In contrast to the original assumption that GSH levels need first to be depleted by $\geq 70 \%$ before adducts begin being formed [9], it is now evident that APAP protein adducts form immediately $[28,30]$. In fact, even after therapeutic doses, which cause only very minor and temporary GSH loss, protein adducts can be detected in the liver and in plasma in mice [30] and humans [31]. A difference between rodents and humans appears to be a higher overall protein adduct generation in human hepatocytes and a more delayed formation of mitochondrial adducts [28] compared to rodents $[30,32]$. Oxidant stress and mitochondrial dysfunction, detected as breakdown of the mitochondrial membrane potential, is followed by cell content release or propidium iodide uptake, indicating necrotic cell death $[28,29]$. APAP also caused JNKactivation in the cytosol of primary human hepatocytes by $6 \mathrm{~h}$; pJNK translocation to mitochondria was observed by $15 \mathrm{~h}$ [28]. In contrast, JNK activation and mitochondrial pJNK translocation occurs in mice within $1-2 \mathrm{~h}[14,32]$. However, APAP did not cause JNK activation in HepaRG cells [28] despite mitochondrial dysfunction and oxidant stress being central to the mechanism of cell death in this cell type [29]. A JNK inhibitor only partially reduced cell death in primary human hepatocytes suggesting that human cells are less dependent on the JNK amplification loop than mouse hepatocytes [28].
In support of the findings with primary cells, evidence for mitochondrial dysfunction was also observed in APAP overdose patients where the release of biomarkers for mitochondrial damage, such as mitochondrial DNA (mtDNA), the mitochondrial matrix enzyme glutamate dehydrogenase and the indirect mitochondrial damage biomarker nuclear DNA (nDNA) fragments, could be measured in plasma [5] (fig. 2). The fact that these biomarkers are not released after furosemide overdose in mice despite the release of alanine aminotransferase (ALT) and development of extensive necrosis suggests these biomarkers are specific for mitochondrial damage and not just cell death [5]. Patients with more mitochondrial damage are less likely to survive, demonstrating that mitochondrial dysfunction is central to the mechanisms of APAP hepatotoxicity in humans [33].

\section{Mechanistic and Predictive Biomarkers of APAP \\ Hepatotoxicity and Acute Liver Failure}

In addition to the above-mentioned mitochondria damage biomarkers mtDNA, glutamate dehydrogenase and nDNA, acylcarnitines were found to be elevated after APAP overdose in mice $[34,35]$. Carnitine is used to shuttle long-chain fatty acids into mitochondria for degradation. Thus, an increase in plasma acylcarnitines, even in the absence of ALT release, reflects mitochondrial dysfunction in hepatocytes [35]. However, no significant increase in plasma acylcarnitine levels was observed in human APAP overdose patients with injury [35]. The reason seems to be at least in part that all patients are treated very early with NAC, which can suppress acylcarnitine release [35]. The likely explanation for this effect is that any NAC not used for GSH synthesis is degraded to form Krebs cycle intermediates, which support mitochondrial energetics [8]. Other biomarkers, such as miR-122 [36], full-length cytokeratin-18 [37], high-mobility group box-1 (HMGB1) protein [37] and argininosuccinate synthetase [38], have all been shown to be highly sensitive for cell damage and necrosis in both animals and APAP overdose patients. These biomarkers are superior to ALT as an indicator of cell injury, i.e. these biomarkers can be detected earlier and, due to a shorter half-life in plasma, are better indicators of acute cell injury [39]. In addition, a panel of miRNAs was shown to be specific for APAP-induced cell death compared to liver injury caused by ischemic hepatitis [40]. In contrast to ALT, most of these biomarkers allow the prediction of whether a patient is likely to survive or will develop acute liver failure and may need a transplant to survive $[33,37,41]$. A limitation of virtually all the 


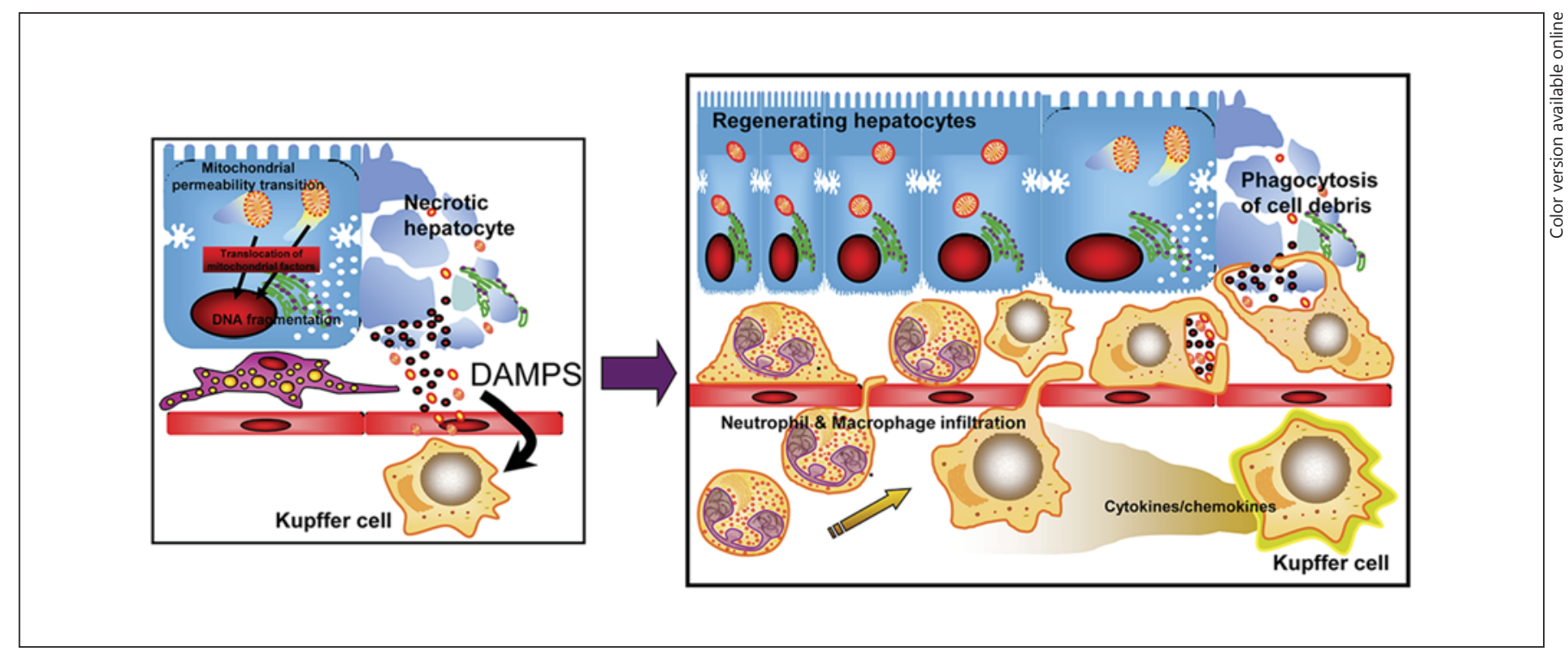

Fig. 2. APAP-induced sterile inflammatory response promotes regeneration. During APAP-induced necrotic cell death a number of cellular components are released, including nDNA fragments, formyl peptides and HMGB1, which can act as DAMPs. DAMPs activate resident liver macrophages (Kupffer cells) via Toll-like receptors. In the regeneration and repair phase, cytokine and che- mokine formation by activated Kupffer cells results in activation, hepatic recruitment and transmigration of neutrophils and macrophages into the damaged tissue to facilitate the removal of dead cells and activation of regenerative pathways. Adapted from Jaeschke et al. [42]. biomarkers evaluated so far is that, although within a large group the biomarker can predict outcome, the substantial overlap in the individual values between groups makes it difficult to rely on the absolute value of any single biomarker [39]. However, several biomarkers could be combined as part of a panel of biomarkers to predict patient outcome, as was used for a mitochondrial damage biomarker [33].

\section{Innate Immune Response and APAP Hepatotoxicity in Rodents and Humans}

Necrotic cell death as observed after APAP overdose in mice and humans causes an innate immune response by release of damage-associated molecular patterns (DAMPs), such as HMGB1, mtDNA and nDNA fragments, etc. [42, 43] (fig. 2). DAMPs bind to various Tolllike receptors, especially on macrophages, and trigger proinflammatory cytokine and chemokine formation. Interleukin-1 $\beta$ (IL-1 $\beta$ ) has received special attention because its formation is not only dependent on the transcriptional activation of the gene, but also on a posttranslational cleavage by caspase-1 [43]. For its activation, caspase- 1 requires the assistance of several scaffolding proteins of the Nod-like receptor family, pyrin domain-containing 3 (NLRP3) inflammasome. The purinergic receptor $\mathrm{P} 2 \mathrm{X} 7$, when stimulated by DAMPs like adenosine triphosphate, can activate the NLRP3 inflammasome [43]. Although there is clear evidence for inflammasome activation and IL- $1 \beta$ formation during APAP overdose in mice $[20,44]$, the pathophysiological relevance of this mediator and the inflammasome is controversial $[20,45]$. The main reason why IL- $1 \beta$ and the inflammasome are likely of very limited importance for the pathophysiology is the fact that the amount of IL- $1 \beta$ being formed is orders of magnitude below levels that would be required to activate immune cells [20]. In addition, mice which lack the IL-1 receptor or any component of the NLRP3 inflammasome are not protected against APAP hepatotoxicity [20, 45]. Furthermore, caspase-1 gene knockout mice or pancaspase inhibitors, which effectively prevented the processing of pro-IL- $1 \beta$ to the active cytokine, did not affect APAP toxicity $[20,45]$. Because IL-1 receptors cannot trigger cell death directly, the main mechanism by which IL- $1 \beta$ could affect APAP-induced liver injury would be to activate inflammatory cells such as neutrophils. Although neutrophils are recruited during the injury phase of APAP hepatotoxicity, a large number of different inter- 
ventions against neutrophils failed to reduce liver injury [reviewed in 42]. The conclusions of a few studies that suggested an aggravation of APAP-induced liver injury by neutrophils have to be questioned due to off-target effects [42]. In fact, some of the most convincing evidence against a role of neutrophils is the observation that circulating and liver-derived neutrophils are not activated during the initial phase of liver injury [46]. In addition, mice deficient in NADPH oxidase, the enzyme responsible for reactive oxygen formation by phagocytes, are not protected against APAP toxicity [42, 47]. Thus, neither neutrophils nor the resident macrophages of the liver (Kupffer cells) contribute to the oxidant stress or injury induced by APAP overdose [42, 47]. In contrast, Kupffer cells actually attenuate the injury through formation of IL-10 [48]. IL-10 limits the generation of proinflammatory cytokines, which may aggravate liver injury by inducing the inducible form of nitric oxide synthase and promoting the mitochondrial oxidant stress [48]. On the other hand, recruitment of neutrophils and macrophages into the area of necrosis is essential for removing cellular debris and promoting regeneration [49]. Taken together, the release of DAMPs by necrotic cells triggers an innate immune response with late activation and hepatic recruitment of neutrophils and macrophages (fig. 2). The majority of the experimental evidence supports the hypothesis that the inflammatory cells are essential for regeneration rather than contribute to the injury in mice [42].

An innate immune response is also observed in humans during APAP-induced liver injury and acute liver failure $[47,50]$. However, there is little evidence for neutrophil activation before and during the peak of liver injury [47]. In contrast, during periods of declining serum ALT levels, i.e. after the active cell death is over, neutrophil activation, as indicated by priming for reactive oxygen formation and the enhanced capacity for phagocytosis, has been observed [47]. As all the patients recovered, the enhanced activation of neutrophils correlated with the regeneration phase [47]. These data support the hypothesis that, similar to the mouse model, neutrophils contribute to the recovery after APAP hepatotoxicity in humans. In addition, hepatic macrophages in livers of APAP-induced liver injury patients are derived from resident macrophages and from circulating monocytes [50]. The expression of the chemokine receptor CCR2 on these cells and the formation of CCL2 indicate that, similar to mice [49], the chemokine CCL2 is responsible for hepatic monocyte recruitment in patients [50]. However, the environment in the liver in the presence of he- patic macrophages is characterized by anti-inflammatory cytokine formation and the expression of proliferation markers [50]. Together, these findings support the hypothesis that hepatic macrophages contribute to the regeneration of the damaged tissue in patients [50] (fig. 2).

\section{Summary and Conclusions}

APAP overdose is the most frequent cause of druginduced liver injury and acute liver failure in Western countries. The mechanisms, which apply to the mouse model as well as to the human pathophysiology, include the formation of a reactive metabolite, which depletes GSH and triggers mitochondrial dysfunction with oxidant stress, JNK activation, $\mathrm{nDNA}$ fragmentation and the MPT, resulting in cellular necrosis. The clinical antidote NAC was developed based on these mechanisms. Although NAC is highly effective when given within $8 \mathrm{~h}$ of the overdose, the efficacy is diminished when treatment is delayed. Further studies of the later phase of the injury and the transition to regeneration are needed to identify therapeutic targets that would benefit late-presenting patients. In addition, an increasing number of biomarkers are being identified that help in understanding the mechanisms of liver injury in patients and will also eventually allow prediction at the time of admission of whether a patient is going to recover or will need a liver transplant to survive.

\section{Acknowledgements}

Work in the author's laboratory is supported in part by $\mathrm{Na}$ tional Institutes of Health Grants AA12916 and DK070195, by grants from McNeil Inc. and Gilead Inc., and by grants from the National Center for Research Resources (5P20RR021940-07) and the National Institute of General Medical Sciences (8 P20 GM103549-07) from the National Institutes of Health.

\section{Disclosure Statement}

The author has no conflicts of interest to disclose. 


\section{References}

$>1$ Lee WM: Acute liver failure. Semin Respir $>16$ Kon K, Kim JS, Uchiyama A, Jaeschke H, LeCrit Care Med 2012;33:36-45.

$>2$ Fontana RJ: Pathogenesis of idiosyncratic drug-induced liver injury and clinical perspectives. Gastroenterology 2014;146:914928.

$>3$ Dart RC, Bailey E: Does therapeutic use of $>17$ acetaminophen cause acute liver failure? Pharmacotherapy 2007;27:1219-1230.

4 Larson AM: Acetaminophen hepatotoxicity. Clin Liver Dis 2007;11:525-548.

5 McGill MR, Sharpe MR, Williams CD, Taha $\mathrm{M}$, Curry SC, Jaeschke H: The mechanism underlying acetaminophen-induced hepatotoxicity in humans and mice involves mitochondrial damage and nuclear DNA fragmentation. J Clin Invest 2012;122:1574-1583.

6 Smilkstein MJ, Knapp GL, Kulig KW, Rumack BH: Efficacy of oral N-acetylcysteine in the treatment of acetaminophen overdose. N Engl J Med 1988;319:1557-1562.

$\checkmark 7$ Corcoran GB, Racz WJ, Smith CV, Mitchell JR: Effects of N-acetylcysteine on acetaminophen covalent binding and hepatic necrosis in mice. J Pharmacol Exp Ther 1985;232:864872 .

$>8$ Saito C, Zwingmann C, Jaeschke H: Novel mechanisms of protection against acetaminophen hepatotoxicity in mice by glutathione and N-acetylcysteine. Hepatology 2010;51: 246-254.

9 Mitchell JR, Jollow DJ, Potter WZ, Gillette JR, Brodie BB: Acetaminophen-induced hepatic necrosis. IV. Protective role of glutathione. J Pharmacol Exp Ther 1973;187:211-217.

10 McGill MR, Jaeschke H: Metabolism and disposition of acetaminophen: recent advances in relation to hepatotoxicity and diagnosis. Pharm Res 2013;30:2174-2187.

-11 Jaeschke H, McGill MR, Ramachandran A: Oxidant stress, mitochondria, and cell death mechanisms in drug-induced liver injury: lessons learned from acetaminophen hepatotoxicity. Drug Metab Rev 2012;44:88-106.

-12 Kon K, Kim JS, Jaeschke H, Lemasters JJ: Mitochondrial permeability transition in acetaminophen-induced necrosis and apoptosis of cultured mouse hepatocytes. Hepatology 2004;40:1170-1179.

13 Gujral JS, Knight TR, Farhood A, Bajt ML, Jaeschke H: Mode of cell death after acetaminophen overdose in mice: apoptosis or oncotic necrosis? Toxicol Sci 2002;67:322328.

-14 Han D, Dara L, Win S, Than TA, Yuan L, Abbasi SQ, Liu ZX, Kaplowitz N: Regulation of drug-induced liver injury by signal transduction pathways: critical role of mitochondria. Trends Pharmacol Sci 2013;34:243-253.

15 Saito C, Lemasters JJ, Jaeschke H: c-Jun Nterminal kinase modulates oxidant stress and peroxynitrite formation independent of inducible nitric oxide synthase in acetaminophen hepatotoxicity. Toxicol Appl Pharmacol 2010;246:8-17. masters JJ: Lysosomal iron mobilization and induction of the mitochondrial permeability transition in acetaminophen-induced toxicity to mouse hepatocytes. Toxicol Sci 2010;117: 101-108.

17 Ramachandran A, McGill MR, Xie Y, Ni HM, Ding WX, Jaeschke H: Receptor interacting protein kinase 3 is a critical early mediator of acetaminophen-induced hepatocyte necrosis in mice. Hepatology 2013;58:2099-2108.

Zhang YF, He W, Zhang C, Liu XJ, Lu Y, Wang $\mathrm{H}$, Zhang ZH, Chen X, Xu DX: Role of receptor interacting protein (RIP) 1 on apoptosis-inducing factor-mediated necroptosis during acetaminophen-evoked acute liver failure in mice. Toxicol Lett 2014;225:445453.

19 Jaeschke H, Cover C, Bajt ML: Role of caspases in acetaminophen-induced liver injury. Life Sci 2006;78:1670-1676.

20 Williams CD, Farhood A, Jaeschke H: Role of caspase- 1 and interleukin- $1 \beta$ in acetaminophen-induced hepatic inflammation and liver injury. Toxicol Appl Pharmacol 2010;247: 169-178.

21 Ding WX, Yin XM: Mitophagy: mechanisms, pathophysiological roles, and analysis. Biol Chem 2012;393:547-564.

22 Ni HM, Bockus A, Boggess N, Jaeschke H, Ding WX: Activation of autophagy protects against acetaminophen-induced hepatotoxicity. Hepatology 2012;55:222-232.

23 Ni HM, Williams JA, Jaeschke H, Ding WX: Zonated induction of autophagy and mitochondrial spheroids limits acetaminopheninduced necrosis in the liver. Redox Biol 2013; 1:427-432.

24 Nagy G, Kardon T, Wunderlich L, Szarka A, Kiss A, Schaff Z, Bánhegyi G, Mandl J: Acetaminophen induces ER dependent signaling in mouse liver. Arch Biochem Biophys 2007; 459:273-279.

25 Tolson JK, Dix DJ, Voellmy RW, Roberts SM: Increased hepatotoxicity of acetaminophen in Hsp70i knockout mice. Toxicol Appl Pharmacol 2006;210:157-162.

26 Saito C, Yan HM, Artigues A, Villar MT, Farhood A, Jaeschke H: Mechanism of protection by metallothionein against acetaminophen hepatotoxicity. Toxicol Appl Pharmacol 2010;242:182-190.

$27 \mathrm{Du}$ K, David Williams C, McGill MR, Jaeschke H: Lower susceptibility of female mice to acetaminophen hepatotoxicity: role of mitochondrial glutathione, oxidant stress and c-jun N-terminal kinase. Toxicol Appl Pharmacol 2014;218:58-66.

28 Xie Y, McGill MR, Dorko K, Kumer SC, Schmitt TM, Forster J, Jaeschke H: Mechanisms of acetaminophen-induced cell death in primary human hepatocytes. Toxicol Appl Pharmacol 2014;279:266-274.
29 McGill MR, Yan HM, Ramachandran A, Murray GJ, Rollins DE, Jaeschke H: HepaRG cells: a human model to study mechanisms of acetaminophen hepatotoxicity. Hepatology 2011;53:974-982.

30 McGill MR, Lebofsky M, Norris HR, Slawson MH, Bajt ML, Xie Y, Williams CD, Wilkins DG, Rollins DE, Jaeschke H: Plasma and liver acetaminophen-protein adduct levels in mice after acetaminophen treatment: dose-response, mechanisms, and clinical implications. Toxicol Appl Pharmacol 2013;269:240 249.

31 Heard KJ, Green JL, James LP, Judge BS, Zolot L, Rhyee S, Dart RC: Acetaminophen-cysteine adducts during therapeutic dosing and following overdose. BMC Gastroenterol 2011;11:20.

32 McGill MR, Williams CD, Xie Y, Ramachandran A, Jaeschke H: Acetaminophen-induced liver injury in rats and mice: comparison of protein adducts, mitochondrial dysfunction, and oxidative stress in the mechanism of toxicity. Toxicol Appl Pharmacol 2012;264:387394.

33 McGill MR, Staggs VS, Sharpe MR, Lee WM, Jaeschke H, Acute Liver Failure Study Group: Serum mitochondrial biomarkers and damage-associated molecular patterns are higher in acetaminophen overdose patients with poor outcome. Hepatology 2014;60:13361345.

-34 Chen C, Krausz KW, Shah YM, Idle JR, Gonzalez FJ: Serum metabolomics reveals irreversible inhibition of fatty acid $\beta$-oxidation through the suppression of PPAR $\alpha$ activation as a contributing mechanism of acetaminophen-induced hepatotoxicity. Chem Res Toxicol 2009;22:699-707.

35 McGill MR, Li F, Sharpe MR, Williams CD, Curry SC, Ma X, Jaeschke H: Circulating acylcarnitines as biomarkers of mitochondrial dysfunction after acetaminophen overdose in mice and humans. Arch Toxicol 2014;88: 391-401.

36 Starkey Lewis PJ, Dear J, Platt V, Simpson KJ, Craig DG, Antoine DJ, French NS, Dhaun N, Webb DJ, Costello EM, Neoptolemos JP, Moggs J, Goldring CE, Park BK: Circulating microRNAs as potential markers of human drug-induced liver injury. Hepatology 2011; 54:1767-1776.

37 Antoine DJ, Jenkins RE, Dear JW, Williams DP, McGill MR, Sharpe MR, Craig DG, Simpson KJ, Jaeschke H, Park BK: Molecular forms of HMGB1 and keratin-18 as mechanistic biomarkers for mode of cell death and prognosis during clinical acetaminophen hepatotoxicity. J Hepatol 2012;56:1070-1079.

38 McGill MR, Cao M, Svetlov A, Sharpe MR Williams CD, Curry SC, Farhood A, Jaeschke $\mathrm{H}$, Svetlov SI: Argininosuccinate synthetase as a plasma biomarker of liver injury after acetaminophen overdose in rodents and humans. Biomarkers 2014;19:222-230. 
39 McGill MR, Jaeschke H: Mechanistic biomarkers in acetaminophen-induced hepatotoxicity and acute liver failure: from preclinical models to patients. Expert Opin Drug Metab Toxicol 2014;10:1005-1017.

40 Ward J, Kanchagar C, Veksler-Lublinsky I, Lee RC, McGill MR, Jaeschke H, Curry SC, Ambros VR: Circulating microRNA profiles in human patients with acetaminophen hepatotoxicity or ischemic hepatitis. Proc Natl Acad Sci USA 2014;111:12169-12174.

41 Woolbright BL, McGill MR, Staggs VS, Winefield RD, Gholami P, Olyaee M, Sharpe MR, Curry SC, Lee WM, Jaeschke H, the Acute Liver Failure Study Group: Glycodeoxycholic acid levels as prognostic biomarker in acetaminophen-induced acute liver failure patients. Toxicol Sci 2014;142:436-444.

42 Jaeschke H, Williams CD, Ramachandran A, Bajt ML: Acetaminophen hepatotoxicity and repair: the role of sterile inflammation and innate immunity. Liver Int 2012;32:8-20.
43 Kubes P, Mehal WZ: Sterile inflammation in the liver. Gastroenterology 2012;143:11581172 .

44 Imaeda AB, Watanabe A, Sohail MA, Mahmood S, Mohamadnejad M, Sutterwala FS, Flavell RA, Mehal WZ: Acetaminophen-induced hepatotoxicity in mice is dependent on Tlr9 and the Nalp3 inflammasome. J Clin Invest 2009;119:305-314.

45 Williams CD, Antoine DJ, Shaw PJ, Benson C, Farhood A, Williams DP, Kanneganti TD, Park BK, Jaeschke H: Role of the Nalp3 inflammasome in acetaminophen-induced sterile inflammation and liver injury. Toxicol Appl Pharmacol 2011;252:289-297.

46 Williams CD, Bajt ML, Farhood A, Jaeschke $\mathrm{H}$ : Acetaminophen-induced hepatic neutrophil accumulation and inflammatory liver injury in CD18-deficient mice. Liver Int 2010; 30:1280-1292.
47 Williams CD, Bajt ML, Sharpe MR, McGill MR, Farhood A, Jaeschke H: Neutrophil activation during acetaminophen hepatotoxicity and repair in mice and humans. Toxicol Appl Pharmacol 2014;275:122-133.

48 Bourdi M, Masubuchi Y, Reilly TP, Amouzadeh HR, Martin JL, George JW, Shah AG, Pohl LR: Protection against acetaminopheninduced liver injury and lethality by interleukin 10: role of inducible nitric oxide synthase. Hepatology 2002;35:289-298.

49 You Q, Holt M, Yin H, Li G, Hu CJ, Ju C: Role of hepatic resident and infiltrating macrophages in liver repair after acute injury. Biochem Pharmacol 2013;86:836-843.

50 Antoniades CG, Quaglia A, Taams LS, Mitry RR, Hussain M, Abeles R, Possamai LA, Bruce M, McPhail M, Starling C, Wagner B, Barnardo A, Pomplun S, Auzinger G, Bernal W, Heaton N, Vergani D, Thursz MR, Wendon J: Source and characterization of hepatic macrophages in acetaminophen-induced acute liver failure in humans. Hepatology 2012;56: 735-746. 